\title{
Come comincia la storia (in Italia): Roma sentimentale di Diego Angeli, 1900
}

\author{
Andrea CORTELlessa \\ Università Roma Tre
}

\begin{abstract}
Nell'ambito della ricca e complessa ricezione di Bruges-la-Morte, atto di nascita del fototesto europeo pubblicato da Georges Rodenbach nel I892, un episodio in apparenza minore, ma curioso, è Roma sentimentale di Diego Angeli, poligrafo sodale di d'Annunzio ma vicino anche alla couche "crepuscolare", testo uscito nel 1900 presso lo stesso editore che pubblicherà nel 1907 la prima traduzione italiana del romanzo di Rodenbach. A differenza di altri spin-off di quegli anni, il testo di Angeli è corredato da un apparato di immagini (fotografie riprodotte su incisioni) e si può forse considerare, allora, il primo fototesto italiano.
\end{abstract}

Keywords: fototesto, Georges Rodenbach, crepuscolarismo, Marino Moretti, Fausto Maria Martini, Diego Angeli, immagine di Roma, Baedeker, plagio.

Vediamo solamente quello che vedono gli altri. Le migliaia di persone che sono state qui in passato, quelle che verranno in futuro. [...] Un'esperienza religiosa, in un certo senso, come ogni forma di turismo. [...] Non possiamo uscire dall'aura. Ne facciamo parte. Siamo qui, siamo ora.

Don DeLillo, Rumore bianco

Uno dei problemi di metodo che si incontrano nella ricostruzione storica dell'iconotesto fotografico consiste nel fatto che, prima degli anni Novanta del Novecento, si fa fatica a impiegare i tradizionali protocolli dei "generi letterari": troppo intermittente e subalterna essendo la sua tradizione, fatta di episodi eminenti (da Nadja di Breton all'Abicì della guerra di Brecht) che magari hanno fatto scuola, sì, ma per aspetti diversi dalla loro compagine verbovisiva. Sicché gli isolati interpreti di questa tradizione paiono doversela reinventare ogni volta (e solo per via indiziaria, di volta in volta, si potranno indurre legami con gli episodi che li hanno preceduti): il che non sarà privo di conseguenze sulla sua ricchezza e imprevedibilità, motivo certo non minore del suo fascino. È una tradizione "carsica", insomma, quella dell'iconotesto fotografico: che ha trovato una sua legittimazione, e dunque un'istituzione, solo alla fine del secolo, il Novecento, che ha attraversato sin dall'inizio. E anzi, prima del suo inizio: se è vero che una convenzione sinora indiscussa (cfr. Cometa 20I6: 69; Albertazzi 2017: 97-98; qualche dubbio in più in Carrara 2020: 89-I09) colloca la fondazione di questo "non-genere" nel romanzo Bruges-la-Morte, pubblicato dal poeta simbolista belga Georges 
Rodenbach nel I892, prima a puntate sul quotidiano parigino Le Figaro, poi in volume presso l'editore Flammarion con l'aggiunta di un paio di capitoli, dell'Avvertenza iniziale e soprattutto di trentacinque fotografie della città (realizzate ritoccando delle cartoline turistiche, dalle quali vennero asportate le didascalie).

Significativamente, anche la fortuna di Bruges-la-Morte è accidentata, dilazionata, "carsica": con momenti di affollata recrudescenza e lunghi periodi di "latenza", in cui resta attivo solo come "libro segreto". Sin dall'inizio poi, e altrettanto sintomaticamente, è stata una fortuna, come si dice oggi, "crossmediale": che si estende, cioè, ben al di là dell'ambito strettamente letterario. Al di là del contrastato riconoscimento di Rodenbach da parte dei letterati suoi compatrioti (o meglio, della sua stessa lingua), o del successo economico del suo astuto editore, Bruges-la-Morte in pochi anni diventa così un libro di culto. Lo stesso Rodenbach ne allestisce una riduzione teatrale, dal titolo Le Mirage, ma non farà in tempo a vederla in scena (una nemesi, se si pensa che l'incontro "fatale" fra Hugues Viane e Jane Scott, nell'intreccio del I892, si era svolto appunto nel teatro di Bruges, dove la donna francese - a dispetto del nome anglosassone - partecipa come ballerina a una messa in scena di Robert le Diable di Meyerbeer), perché lui muore (a soli 43 anni, per i postumi di una banale operazione d'appendicite) il giorno di Natale del I898, mentre la première di Le Mirage si terrà solo nel I9oo.

Ancora più popolare si rivelerà l'ulteriore riduzione che del dramma ${ }^{1}$ realizza il compositore tedesco Erich Wolfgang Korngold, allievo di Alexander von Zemlinsky e protégé di Gustav Mahler: l'opera musicale Die tote Stadt, 'La città morta', composta nel I9I6 ma andata in scena solo dopo la fine della Grande Guerra, nel '20. Sarà questo il maggior successo, forse, del teatro musicale del Novecento (Puccini a parte), ed è infatti tuttora in repertorio. Korngold, ebreo, dovrà poi fuggire dai nazisti e rifugiarsi negli Stati Uniti, dove diverrà uno dei più apprezzati compositori di colonne sonore per l'industria hollywoodiana. E proprio a Hollywood - tramite il noir dei mestieranti Boileau e Narcejac, D'entre les morts - la trama di Rodenbach incontra il capitolo verticale, nonché di gran lunga il più fortunato, della sua sorprendente fortuna crossmediale: Vertigo (ossia, in Italia, La donna che visse due volte) di Alfred Hitchcock, che esce nel $1958^{2}$.

A poca distanza dalla pubblicazione originaria di Bruges-la-Morte, invece, si era prodotto - come nell'Autunno del Medioevo - un curioso "gemellaggio" tra le Fiandre e l'Italia. Il "culto" per Bruges-la-Morte e il suo autore era stato infatti particolarmente vivace fra i poeti della couche piemontese-romagnolo-romana che un critico allora non simpatizzante, Giuseppe Anto-

I E non del romanzo, se è vero che riporta le sottili varianti all'inventio di Bruges-la-Morte apportate da Rodenbach in Le Mirage (cfr. Nieddu 20I2).

2 Rinvio a Cortellessa (2018). 
nio Borgese, aveva definito «crepuscolari» (cfr. Violato 1965, Livi 1974, Farini 1992). Dopo la morte precoce Rodenbach diventa, per questi autori, un vero maestro: in particolare nell'ambiente romano, quello cioè dal più spiccato penchant simbolista, capitanato da un altro poeta destinato a precocissima morte, Sergio Corazzini ${ }^{3}$. Non solo vengono citate, e talvolta plagiate, le raccolte poetiche di Rodenbach (e di altri poeti simbolisti belgi a lui più o meno vicini, come Émile Verhaeren e Maurice Maeterlinck), magari mediate dalla circolantissima antologia Poètes d'Aujourd'hui di Bever e Léautaud (per questa generazione un po' il corrispettivo del Canzoniere per i petrarchisti del Cinquecento, nell'umorosa sintesi di Sanguineti 1984: 26-27), come fanno Corrado Govoni e Aldo Palazzeschi prima di aderire fuggevolmente, ma incendiariamente, al Futurismo di Marinetti ${ }^{4}$; ci sono pure scrittori che si rifanno al singolare mix di decadentismo, religiosità e impegno sociale e politico che caratterizza la personalità di Rodenbach (si pensi al popolarissimo Antonio Fogazzaro, il cui romanzo più discusso, Il Santo del 1905 che verrà messo all'Indice da Santa Romana Chiesa, comincia e si conclude proprio a Bruges, la "grande mistica morta»). C'è anche Gabriele d'Annunzio, che scrive la tragedia La città morta, andata in scena a Parigi l'anno della morte di Rodenbach, il I898; tuttavia l'Imaginifico, spregiudicato plagiario della letteratura universale, in questo caso dal mito di Bruges - in tal senso già codificato da Baudelaire - riprende, forse, solo il titolo ${ }^{5}$ (a imagery e Stimmung della poesia belga aveva guardato semmai nel Poema paradisiaco, pubblicato nel I893).

Ci sono poi scrittori che a Bruges si recano in pellegrinaggio - non si può dire diversamente - per vedere dal vero i paesaggi del romanzo. È il caso del romagnolo Marino Moretti (I885-1979), che all'inizio del Novecento aveva fatto parte a sua volta del cenacolo "crepuscolare" ${ }^{6}$. Anni in cui, sarà bene

3 Si vedano gli informatissimi Villa (1999) e Comberiati (2014). Una vivace ricostruzione di prima mano, ancorché non troppo fededegna, è quella di un comprimario del gruppo che però svolgerà un ruolo importante nella storia che sto cercando di ricostruire: si veda la prima parte delle memorie (da lui però definite «romanzo») di Fausto Maria Martini (I886-I93I), Si sbarca a New York (1930).

4 Un titolo esemplare di Govoni, Armonia in grigio et in silenzio del 1903, riprende pari pari l'imagery della Bruges di Rodenbach: le beghine, i chiostri, appunto il silenzio e persino il grigio, che è il colore delle vesti punitive delle beghine nonché quello delle fotografie nel libro... (cfr. Govoni 1903, 1989, 1992).

5 La storia riguarda una campagna di scavi archeologici in Grecia; anche la storia di Rodenbach si può considerare in un certo senso "archeologica", ma qui d'Annunzio guarda piuttosto alla moda internazionale, all'epoca non meno fiammeggiante, degli scavi appunto in Grecia e in Asia minore: al I872 risaliva la clamorosa scoperta di Troia da parte di Heinrich Schliemann.

6 Prezioso il contributo di Livi 2002, che all'inizio ricorda anche i lemmi, del mito di Bruges, che precedono Rodenbach (fra Wordsworth, Baudelaire e Longfellow). Cfr. pure Bigongiari (I977), Farini (2002) e Guglielminetti (2002). 
ricordarsene, tutti questi giovani poeti infatuati di Rodenbach erano senza il becco d'un quattrino, sicché Bruges potevano visitarla solo virtualmente, magari proprio nelle fotografie riprodotte sulle pagine di Bruges-la-Mor$t e^{7}$ : ideale Baedeker che creava, se così si può dire, un luogo dell'anima per procura (infatuazione che ha per paradigma la «Ferrara come Bruges» di Govoni) ${ }^{8}$. Anche Moretti cioè, come Gozzano in India, è «uno di questi voyageurs "autour de sa chambre"»; anche lui in un primo momento quel «luogo topico della memoria» che è Bruges lo «ha visto e amato prima di vederlo» e lo ha percorso, sentimentalmente, «come una favolosa Carte du Tendre», per dirla con un bel saggio di Piero Bigongiari (1977: 45). E anzi quel filtro cartaceo, non senza snobistico compiacimento, una volta Moretti giunse a rivendicarlo, annotando le Poesie scritte col lapis - che dal mito fiammingo si presentano sature sin quasi al sospetto della parodia - nell'autoantologia del I919, Poesie 1905-I9I4: la lirica La domenica di Bruggia «fu scritta non già dopo la lettura dei poeti Rodenbach e Max Elskamp come sospettarono i critici, a suo tempo; ma dopo aver letto alcune note di viaggio della signorina Marianna Cavalieri nella rivista La Donna (I908)» (Moretti I9I9: 284) ${ }^{9}$.

All'altezza del I9ıo, nella Domenica di Bruggia appunto, rintoccava il refrain «laggiù in fondo, nelle amare / solitudini ove anch'io / sarò un dì col mio desio / implacabile di andare... // sì, di andare fino a Bruggia / fino al vecchio beghinaggio / per vedere un paesaggio / lagunare che si aduggia» (I33). Negli anni Venti da un pezzo ha smesso di scrivere poesie, Moretti; pubblica invece romanzi con Mondadori che, ancorché non sia la corazzata di oggi, può già consentire ai suoi autori un tenore di vita un po' più dignitoso della bohème, ai limiti della fame, sperimentata in passato. Così a quel punto Moretti finalmente può realizzare un sogno a lungo accarezzato: nel I925 visita prima Parigi e poi il Belgio, in compagnia di Filippo de Pisis ferrarese come

7 Ad Antonio Baldini scriverà molto tempo dopo, Moretti, che a quell'altezza per la verità aveva letto solo Maeterlinck; per il resto la Stimmung di Bruges era filtrata, significativamente, $\mathrm{da}$ «qualche articolo di giornale o di rivista, in qualche fotografia o veduta di Bruges-la-morte» (Marino Moretti ad Antonio Baldini, I2 gennaio I950, in Baldini-Moretti (I997: 237), cit. in Livi (2002: I54n)).

8 Il quale nella città fiamminga non mise mai piede ma, ricorda Francesco Targhetta, si dilettava a mandare agli amici cartoline di Bruges trovate sulle bancarelle di Roma (cfr. Livi I984; Targhetta 2008: II7 sgg.; Targhetta 2016).

9 Senza dettagliarlo, il cenno è pure nella nota apposta alla successiva autoantologia Tutte le poesie: «Per questa "Domenica di Bruggia" [...] scritta nel I909, l'autore non poté servirsi che di notizie apprese da una popolare effemeride illustrata. Da ciò l'evidente genericità del paesaggio» (Moretti 1966: II49). Ma proprio la precisione del rinvio bibliografico del 'í9 a qualcuno parve intenzionalmente sviante, se non borgesiana avant la lettre; e invece col suo proverbiale talento di segugio Franco Contorbia ha rintracciato il reportage in questione. Che poi in effetti sono due, entrambi apparsi sul settimanale La Donna appunto, sotto l'equivoco titolo complessivo di Viaggio in Olanda: Il «Buen Retiro», 20 settembre 1908, e Hanse fiamminga, 20 giugno 1909 (cfr. Contorbia 1980, che riproduce per intero il primo dei due articoli). 
Govoni, e non ha motivo di resistere all'attrazione di Bruges ${ }^{10}$. Seguiranno, nella città fiamminga, una serie di ritorni e soggiorni anche prolungati. Al ritorno scrive su questa esperienza un libro curioso, La Casa del Santo Sangue, che pubblica nel 1929 a puntate sul supplemento del Corriere della Sera, anche allora intitolato «La Lettura», e l'anno seguente in volume appunto da Mondadori" ${ }^{\text {II }}$ Il libro si legge a tutti gli effetti come un Bruges-la-Morte reloaded, effetto anche autoironicamente sottolineato (alla protagonista la «sotto-superiora, la vice Grande Dame» del Béguinage si rivolge a un certo punto sprezzante: $« \mathrm{Ah}$, ah, voi siete un'intellettuale! avete letto i romanzi di Rodenbach! siete venuta per Rodenbach!» (Moretti 1930: I23-I24)). Pure la copertina della prima edizione, seppur banalizzata dalle tinte pastello, replica l'immagine canonica, la «joli vue» del Minnewater che separa e congiunge la città "nuova" e mercantile all'hortus conclusus del Béguinage (scorcio nel libro di Rodenbach disegnato da Fernand Khnopff al frontespizio, e riprodotto fotograficamente all'inizio del primo capitolo):

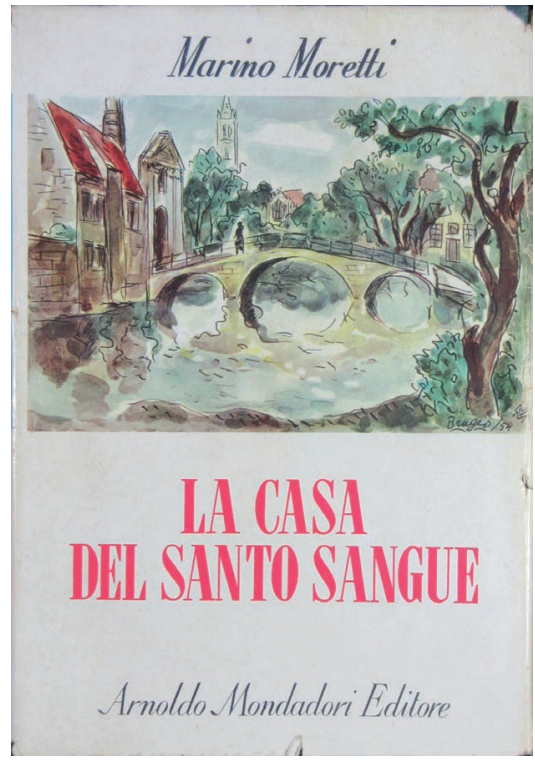

Fig. I: Marino Moretti, La Casa del Santo Sangue (I930), copertina.

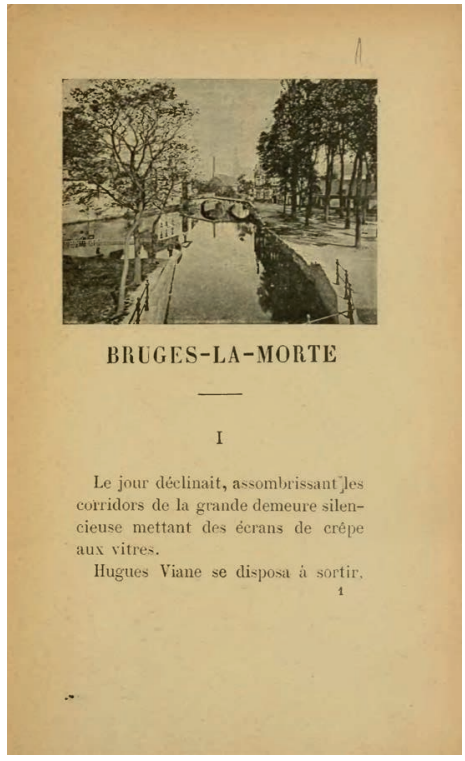

Fig. 2: Georges Rodenbach, Bruges-la-Morte (I892), prima pagina illustrata.

Io Marino Moretti ad Aldo Palazzeschi, I2 giugno I925: «la settimana belga è stata faticosissima e [...] solo Bruges, di quelle città, non mi ha deluso. (Ah, Bruges è indimenticabile!)» (Moretti-Palazzeschi 1999: 472).

II All'indomani del viaggio, sul Corriere della Sera il 2 agosto 1925, Moretti aveva già pubblicato la novella Bruges, "cartone" del romanzo. 
Un mito rivissuto a posteriori dunque; ma anche, ormai, troppo tardi. Non direi, come fa François Livi (2002: 156), che La Casa del Santo Sangue si possa definire «la suprema interpretazione» del «mito di Bruges»; si può ben dire, invece, che ne sia «la conclusione» (per quanto riguarda, almeno, la nostra letteratura). Dai tempi degli “anni Corazzini” quel mito, e non solo dentro Moretti, è appassito da un pezzo. Tanta acqua è passata sotto $\mathrm{i}$ ponti di Bruges: di mezzo ci sono stati l'avanguardia, la guerra, il fascismo. In Italia è cambiato tutto.

La Casa del Santo Sangue non è un testo (almeno esplicitamente) autobiografico; è un romanzo di finzione in cui la giovane Marta Saccomandi, ferita da una serie di disavventure sentimentali, vorrebbe separarsi dal mondo rinchiudendosi nel Béguinage, ma resta poi divisa fra il richiamo dello spirito e quello della carne. Alla fine rinuncerà al suo sogno di purezza monastica, tornerà in Italia e accetterà di divenire madre. Considerando l'omosessualità di Moretti, non si fatica a capire come tutto questo rivestisse per lui un significato allusivo ${ }^{12}$; ma dal punto di vista letterario l'effetto di un simile scioglimento è quello di porre una pietra tombale sul mito di Bruges, sull' infatuazione che era stata la sua e della sua generazione di amici poeti: il suo è un congedo definitivo, in termini interiori, a questa patria dell'anima ${ }^{13}$.

A stregare i poeti crepuscolari era la vita bloccata cantata da Rodenbach: una vita che si guarda dentro ma soprattutto alle spalle, cristallizzata in un ripiegamento depressivo che si rivolge alla religione - è evidente dalla trama del romanzo di Moretti - come a un balsamo privato di ferite intime, non necessariamente spirituali. Il sacrario feticistico di Hugues, che conserva sotto una teca di cristallo la treccia tagliata alla moglie morta (e proprio con quella pone termine ai giorni della nuova compagna Jane, da lui considerata impari a quel modello - almeno sino a quando si ostina a vivere), è allegoria che codifica a perfezione questo stato d'animo. Simile era la Stimmung con la quale in quegli anni veniva vissuta, dagli stessi poeti, la città di Roma: in modo risolutamente antitetico, cioè, a come la nuova Capitale cresceva, allora, tanto disordinatamente quanto euforicamente (basti pensare al Piacere

I2 Del resto dichiara lui stesso nella conclusiva Nota dell'autore: «È un po' un luogo comune parafrasare Flaubert [...], ma io ero allora tentato di trascurare la protagonista per dire a chi me ne chiedeva qualcosa che... "Il beghinaggio sono io"» (Moretti I930: 268).

I3 Cfr. Romagnoli (1977) e Robaey (1993). Un altro decennio a posteriori, Moretti torna sulla vicenda nell'articolo - stavolta esplicitamente autobiografico, occasionato dall'uscita in traduzione fiamminga della Casa del Santo Sangue, titolo italiano però innominato - «Bruges la risvegliata», Corriere della Sera, 25 agosto 1938, che ricorda: «Tornato a Bruges dopo molt'anni, parendomi di tornarvi da vecchio, ho subito la misura di come siamo cambiati io e la città, lo scrittore e la sua ispiratrice. Io ho rinunciato al pallido blasone delle mie origini letterarie, la città ha rifiutato la statua al suo poeta, Giorgio Rodenbach, che le aveva dato con Bruges-la-morte una gloria sospetta. [...] E così Bruges non vuol più essere Bruges-la-morte e l'antico crepuscolare ha il senso preciso della sconfitta d'una scuola letteraria italiana quando legge il titolo di un libro nuovo esposto in vetrina: Bruges-la-réveillée». 
del solito d'Annunzio, che è del I890). La Roma di Corazzini e della sua couche, di contro, si trincera nella sua identità cattolica e nei propri scenari archeologici; snobisticamente evita le scenografie più illustri e consuete compiacendosi invece di esplorarne gli anditi più nascosti e (allora) periferici. E in questa Roma segreta ci imbattiamo nel capitolo che di questa storia è il più segreto.

La prima delle quattro traduzioni italiane di Bruges-la-Morte esce nel 1907. Ne è autore un altro poeta romano di questa cerchia, il non irresistibile Fausto Maria Martini (cfr. Comberiati 20I4: IOO-II3), e la pubblica un editore della sua città, Enrico Voghera, che in quegli anni pubblica anche autori quotati come il citato Fogazzaro, il popolare poeta romanesco Trilussa o il non meno popolare narratore per l'infanzia Yambo (al secolo Enrico de' Conti Novelli da Bertinoro). In una sua collana abbastanza diffusa, la «Piccola collezione Margherita» (che in seguito pubblicherà anche, fra gli altri, De Amicis e Deledda, Serao e Capuana), l'editore Voghera aveva pubblicato qualche anno prima, giusto nel 1900 , il libro di un giornalista di successo a sua volta vicino a Corazzini ma soprattutto amico di d'Annunzio, Diego Angeli (I869-I937; cfr. Veneruso I96I). Già autore di un paio di romanzi, Lïnarrivabile nel I89I e Liliana Vanni nel 190o, apprezzati anche dal severo Luigi Russo (1923:59) e ambientati in «una Roma che rievoca la Bruges dell'ammirato Rodenbach» (Faitrop Porta 1992: 19), ma pure dannunzieggianti anzichenò ${ }^{\text {I4 }}$, in seguito si segnalerà come sapido memorialista della vita culturale di quel tempo ${ }^{15}$.

Il libro s'intitola Roma sentimentale e incontrò un certo successo, se è vero che nel 1904 se ne fece una seconda edizione. È in sostanza un Baedeker, una "guida" della città, che però ne evita le rotte turistiche più consuete per esplorarne, alla maniera appunto crepuscolare, luoghi poco canonici per non dire segreti. Se dunque si presenta come testo "di servizio", merita invece d'essere letterariamente preso in considerazione: almeno secondo gli standard del nostro tempo, ben più inclusivi di quelli di allora nei confronti della non fiction (come oggi, appunto, si è preso a definirla; esempio letterariamente più valido, nonché assai più celebre, è la deliziosa Guida sentimentale di Venezia pubblicata nel 1942 dal poeta e francesista Diego Valeri e tante volte in seguito riproposta; ma appunto ai giorni nostri è, questo, format letterario piuttosto frequente).

Come scrisse in un pezzo deliziosamente snobistico uno che di Angeli era stato amico alla corte di Gegé Primoli, cioè Mario Praz, il fascino che può esercitare Roma sentimentale è quello della «documentazione» di un'epoca precisa del gusto, l'«epoca floreale dannunziana», della quale offre un'epitome ai limiti della «perfezione: la perfezione dei clichés inevitabili» (Praz 1940: 330). Fra l'altro del testo Praz nota un preciso "valore d'uso", non però in chiave

I4 Cfr. D’Anna (1996); D'Anna (I998); Oliva (2002).

I5 Cfr. Angeli (1930); sul giornalista cfr. Oliva (2002) e Contorbia (2007). 
tradizionalmente turistica: «il libro di Angeli vuol essere come una guida ovidiana, piena di discreti suggerimenti circa i luoghi più adatti ai convegni d'amore» (33I). Eppure sintomaticamente Praz (che pure fu pioniere, com'è noto, negli studi sull'imagery letteraria e, in particolare, su quella particolare comunicazione verbovisiva che fu l'emblematica cinque-seicentesca) non fa alcun cenno, in questo suo pezzo, alle immagini contenute in Roma sentimentale (benché, come vedremo, alcune di esse fossero state realizzate dal comune mentore Primoli); a meno che non vi alluda la metafora (o meglio, catacresi) dei «clichés», nel senso di "luoghi comuni".

Della prima edizione di Roma sentimentale (detta «assai rara», già nel '40, anche da un bibliofilo trascendentale come Praz: 329) sono riuscito a vedere una sola copia, nel patrimonio bibliotecario del mio paese, presso il fondo della CASA DEL FASCIO di Bologna (come si può vedere da un timbro rosso squillante al frontespizio, nonché su quasi ogni pagina) ereditato dalla $\mathrm{Bi}$ blioteca dell'Archiginnasio della stessa città; ma se posso offrire qui una scelta di riproduzioni in pulito dal suo impaginato è grazie alla gentilezza di Vega Tescari e della Bibliothèque cantonale et universitaire Lausanne ${ }^{16}$.

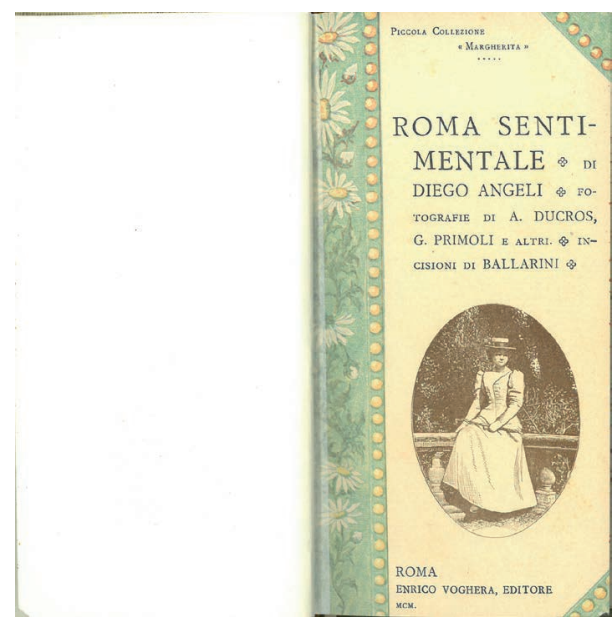

Fig. 3: Diego Angeli, Roma sentimentale, ed. 1900, copertina.

\begin{abstract}
I6 La Bibliothèque publique et universitaire de Neuchâtel conserva una copia della traduzione tedesca del libro, verosimilmente spacciata nel mercato abroad quale Baedeker, della città, in senso stretto (Angeli 1905). Un altro testo di Angeli, pubblicato nello stesso I900 ma più tradizionalmente afferente alla tipologia del Baedeker, è Le chiese di Roma. Angeli rielaborerà e amplierà questi testi in una serie di volumi intitolati semplicemente Roma (Bergamo, Istituto di Arti Grafiche, 1907) riproposti, negli anni Trenta, in una fortunata serie di titoli pubblicati da Treves. Recentissima l'edizione dei suoi Taccuini romani (che a dispetto del titolo sono dipinti di piccolo formato, unica testimonianza della vena pittorica di Angeli), pubblicati in occasione della mostra omonima curata da Silvana Bonfili al Museo di Roma in Trastevere dal I novembre 2019 al 23 febbraio 2020 (Angeli 2020).
\end{abstract}


Laggettivo del titolo fa ovviamente riferimento al celebre Sentimental Journey di Laurence Sterne, spassosissimo romanzo settecentesco tradotto in italiano nientemeno che da Ugo Foscolo: "sentimentale" è il viaggiatore imprevedibile, quello che appunto evita le rotte consuete e procede senza mete fisse, seguendo solo l'andirivieni dei suoi umori, cioè appunto i sentiments a loro volta destati dall'"anima" dei luoghi che percorre. Come dice Angeli dei «luoghi» della città: «ognuno di questi luoghi non aveva forse un'anima che poteva vibrare simultaneamente e armonicamente con l'anima nostra?» (I900: I8). È il paradigma romantico codificato da una celebre pagina di Amiel ("Ogni paesaggio è uno stato d'animo», si legge il 3I ottobre I852 sul suo Diario; I884-1927: I42 ${ }^{17}$ ma modernamente messo a frutto, con l'ausilio del ritrovato fotografico, appunto da Rodenbach ${ }^{18}$. A questo effetto, attentamente pianificato, è volto appunto l'apparato fotografico, come dichiara l'Avvertenza iniziale del suo romanzo: «Poiché gli scenari di Bruges contribuiscono alle vicende, è altrettanto importante riprodurli qui, intercalati tra le pagine» (Rodenbach 1997:3n) ${ }^{19}$.

"Sentimentale", in questo senso, è pure l'apparato iconografico del libro di Angeli, che ne fa a mio parere a tutti gli effetti un iconotesto: dal momento che le immagini non sono, o non si possono dire sempre, vere e proprie "illustrazioni" del testo verbale. Nella pagina che riproduco qui (e la cui foto è riprodotta, come abbiamo visto, sulla copertina del libro), ci troviamo per esempio nel primo di questi luoghi "segreti" della città, Villa Balestra, all'epoca all'estrema periferia Nord della città; ma la signora appoggiata al parapetto potrebbe trovarsi, in effetti, in qualsiasi altro parco di Roma:

I7 Per la circolazione dell'idea di paesaggio "à la Amiel" nella cultura pittorica romana di fine Ottocento, strettamente legata a d'Annunzio, cfr. Lombardi (2003). Interessante sarebbe verificarne la "tenuta" anche nella cultura fotografica "dannunziana" precedente a Primoli: si veda intanto Falzone del Barbarò (1979).

I8 Che riprende alla lettera lo slogan di Amiel: «Ogni città è uno stato d'animo, e basta soggiornarvi un po' perché tale stato d'animo si comunichi, si prolunghi in noi attraverso un fluido che s'inietta e si incorpora con le sfumature dell'aria» (Rodenbach 1997: 53).

I9 La frase dell'Avvertenza deve essere citata dalla curatrice italiana in nota, e non figura nel testo italiano, per il semplice motivo che da questa edizione (come del resto dalle altre quattro apparse nella nostra lingua fra il $1907 \mathrm{e}$ il 20I6) sono proditoriamente tagliate le immagini cui appunto l'autore qui si riferisce (rinvio a Cortellessa 20I8). 


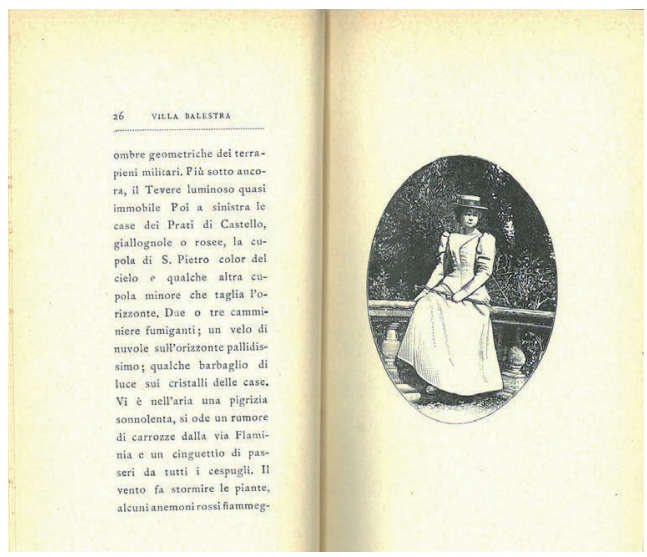

Fig. 4: Diego Angeli, Roma sentimentale, ed. 1900, prima pagina illustrata.

Fra i capitoli successivi si annoverano Villa Borghese, Porta Portese o il Cimitero Inglese: siti della città, in seguito assurti all'immaginario collettivo, che oggi tutto ci paiono meno che "segreti" (il medesimo cortocircuito degusta Praz nel leggere della "scoperta" di San Saba, che parrebbe incongrua «se anche noi non ci ricordassimo che, ancora venticinque anni fa, una visita a San Saba aveva tutto il fascino d'una scoperta»: 1940: 333). Il gusto snobistico-collezionistico di rivelare aspetti inediti di paesaggi celebrati l'aveva pure d'Annunzio, certo; ma in Roma sentimentale è presente altresì una diversa sensibilità, quella ripiegata detta appunto «crepuscolare $»^{20}$, "firma" del resto ostentata a più riprese dall'autore (a San Saba, appunto, si «assapora tutta la dolcezza di quell'ora crepuscolare»: Angeli 1900: 66). Qui si può presumere che la visita avvenga in effetti all'ora del tramonto (come lo sarà quella a Villa Borghese allagata dalla «luce crepuscolare»: 135); ma nell'episodio successivo - ambientato nell'attuale quartiere Prati, oggi per ironia della sorte opulento bastione giudiziario-militare della città - il «paesaggio di miseria e desolazione» di quella che in seguito si sarebbe detta una "borgata" (con tutto l'apparato pasoliniano avanti lettera delle «strade non lastricate», delle «zolle fangose», degli animali liberi di «diguazzare nelle pozze») riceve questa evidente connotazione simbolica: «sembra veramente che in quel luogo debba piovere senza fine e che le vie debbano essere sempre piene di un fango appiccicoso e tenace e che una triste luce di crepuscolo debba

20 Cioè «il 'piccolo mondo' [...] in opposizione al 'grande mondo' [...] come meccanismo di protezione e di rinuncia»: Sanguineti (1984: 30). Peraltro una certa vena dannunziana era tutt'altro che incompatibile, si sa, con la sensibilità "crepuscolare" (cfr. Comberiati 20I4: 66-82). 
rischiarare, in eterno, quelle case di dolore» (68-69; e alla fine si stende un «ultimo crepuscolo»su «quelle case miserabili e tristi»: 73$)^{21}$.

Più in generale, l'itinerario di Angeli indugia con allusiva preferenza nei set d'elezione del repertorio, e diciamo pure della koinè, della poesia crepuscolare. In tal senso, per esempio, il convento di San Bonaventura al Palatino - il cui «triste borgo» circostante, «con le case affumicate e ingrommate, tutte archi, portoncini, sottoscale», cui succede l'accesso al reclusorio vero e proprio la cui «grande calma benefica» offre balsamico rifugio dall'incendio di sensualità dell'estate circostante (86) - è un evidente remix romano del Béguinage originale. Sono «sentiment $i$ misteriosi e profond $i »$ quelli di cui va a caccia il viaggiatore sentimentale, che penetra le «mura [...] inaccessibili alle anime volgari» di Villa Medici, già sede degli exploits del maestro nel Piacere e dintorni: «veramente un luogo di chimere [...], come una oasi ignorata che dia nuovo ristoro ai pellegrini del sogno» (I76-I77). Ma altrettanto connotata è la visita a quell'altro hortus conclusus che è il Bosco Parrasio, sede dell'Accademia dell'Arcadia: «vecchio giardino umido e abbandonato» che si visita, per colmo di stereotipia, «il pomeriggio di una estate dei morti pieno di dolcezza e di languore», e in cui s'incontra «Rosalba», «una donna non più giovane, coi capelli bianchi ma col volto ancor roseo e fresco e la bocca un po' dolente ma di una purezza infantile» (I67-I69). Bocca che, al fin della licenza, mormora ovviamente: «Troppo tardi. Troppo tardi!» (I74).

In ogni caso, quella attribuita a Villa Balestra è la prima delle undici immagini incluse nel testo (cui si aggiunge, al controfrontespizio, il ritratto dell'autore) che, avvertono copertina e frontespizio, sono «fotografie di A. Ducros, G. Primoli e altri», riprodotte nelle «incisioni del prof. Ballarini» ${ }^{22}$ (si

2I Hanno un rilievo a loro volta simbolico, in clausola alla visita a Villa Borghese, le «cose crepuscolari» (piume di gru e di pavoni, e «voli silenziosi» di «farfalle crepuscolari» nel «cielo d'oro», «sciami di petali» sulla superficie del «laghetto increspato», «odori misteriosi»: Angeli I900: 138-139). Esplicita si fa questa dimensione nell'«ultima passeggiata» indirizzata per una volta in una sede ideale che non corrisponde ad alcun luogo specifico, l'«altare del Dio Ignoto» (195 sgg.). Ma del resto parla chiaro, in tal senso, già la scelta di ambientare la maggior parte delle visite verso il crepuscolo: perché (nel capitolo intitolato «Lungo il Tevere») «è verso il tramonto, quando dai prati viene un più intenso odore di fieno e un più grave silenzio sembra discendere dal cielo, che il fiume acquista un aspetto religioso» (159) e ci si inoltra nella notte, «compresi della grandezza di tutte quelle cose piene di mistero» (I65). L'idillio alla marana con l'innominata «amica» che reca «negli occhi la traccia del maleficio lunare» (I65) è il momento in cui il testo più si allontana dall'andamento-Baedeker per tentare l'ascesa all'estasi panica del modello dannunziano.

$22 \mathrm{Al}$ momento non sono riuscito a reperire informazioni su «A. Ducros» (sarebbe curioso se fosse un discendente di Abraham-Louis-Rodolphe Ducros, I748-I8Io, pittore e incisore svizzero celebre appunto per i suoi paesaggi di Roma e dintorni); il «prof. Ballarini» è Ernesto Ballarini (I845-I922), incisore bolognese che a Roma dirigeva all'epoca la scuola d'incisione in intaglio dell'Ospizio di San Michele ed era apprezzato collaboratore di editori importanti come Treves e Sonzogno e di riviste illustrate come L'Italia, da lui stesso fondata. Anche altri titoli della «Piccola Collezione Margherita» sono illustrati da incisioni di Ballarini, ma in quei 
noti che proprio il passaggio litografico consente l'uso della medesima carta in tutta la foliazione del libro, diversamente da quanto si osserva un po' in tutta la tradizione del fototesto prima dell'epifania di Sebald). Sebbene non con la radicalità di Bruges-la-Morte ${ }^{23}$, le immagini non sono giustapposte in modo del tutto lineare, si diceva, rispetto alle descrizioni verbali dei luoghi cui fanno riferimento; il che dipende dal fatto che la rêverie di Roma sentimentale è connotata da una temporalità "mista", a sua volta ambigua e non lineare ("tra i cipressi di Monte Mario», per esempio, si affollano i ricordi e quello messo in scena è in effetti un «ritorno»: «in quello stesso luogo, in quella stessa stagione, con un'altra donna, per ritrovare l'antico sogno giovanile»: Angeli 1900: 3I).

Come si vede, si ripete qui con esattezza il cortocircuito di Rodenbach fra lo stesso luogo e un'altra donna: solo virando d'un sottinteso galante (e un po' malandrino) quella che, nell'originale, è un'allucinazione onirica e macabra. Il ben diverso mood emotivo non deve però farci sfuggire che l'interazione fra testo verbale e fotografia ha lo stesso effetto con intelligenza messo a fuoco da Paul Edwards in Bruges-la-Morte (1997: 40): secondo il quale proprio l'inserimento dell'immagine fotografica, col suo sottinteso di «passeità» (il «noema» dell'«è stato» di Barthes), proietta in una sfera indeterminata e "assoluta", accentuandolo appunto sino all'allucinazione, lo sguardo retrospettivo del testo.

Proprio questa indeterminazione dei luoghi e dei tempi della rappresentazione, e la sottile dissimmetria che ne risulta fra le descrizioni e le immagini, sono caratteri di Roma sentimentale verosimilmente desunti dal precedente di Bruges-la-Morte: del resto vero e proprio libro feticcio, s'è visto, della couche letteraria di Diego Angeli ${ }^{24}$. E allora, se l'ipotesi presente ha qualche

casi più tradizionalmente tratte da disegni al tratto. «G. Primoli» è naturalmente il celebre Giuseppe Napoleone, ma per tutti «Gegé» Primoli (I85I-1927), discendente della famiglia Bonaparte e stella di prima grandezza nella cerchia romana di d'Annunzio: collezionista di tutto il collezionabile, memorialista poligrafo e bon vivant, fu insieme al fratello Luigi anche un pioniere della fotografia d'arte italiana, oggi celebrato per aver tramandato nelle sue lastre la «Roma che se ne va» (cfr. Negro I962 e D'Anna 20I6). Al suo nome è intitolata l'omonima Fondazione, presieduta da Mario Praz dal ' 58 all' 82 (dalla quale dipendono l'importante $\mathrm{Mu}$ seo Napoleonico - di cui primo direttore fu, dal '27 al '37, proprio il nostro Diego Angeli - e appunto un imponente Archivio fotografico).

23 Dove l'oscillazione cronotopica suggerisce un'«essenza», del luogo, «situata al di fuori del tempo»: effetto questo accentuato dalla dislocazione delle immagini, le quali hanno una «relazione indiretta col testo: l'effetto ottenuto ha la funzione di una cesura» (Grojnowski 1995: I3I-I32, traduzione mia).

24 Forse una sphraghis autoriale è da leggersi nel cenno a «una piccola casa, col tetto aguzzo alla maniera fiamminga» intravista nel quartiere di Borgo, al limitare della Basilica di San Pietro (Angeli 1900: 78). Più dubbia l'allusione al Cimitero degli Inglesi, dove sulla tomba di Shelley (visitata naturalmente «in un estremo crepuscolo di inverno, quando un pallido sole filtrava obliquamente tra il colonnato di cipressi») è stato «deposto un mazzo di bucaneve con un dolce nome: Jane. E io mi domandai se veramente non era lo spirito della Magnetic Lady che 
valore, mi pare quanto mai simbolico che il primo iconotesto fotografico in Italia venga pubblicato proprio nel I900: con almeno mezzo secolo di anticipo, dunque, sui primi esemplari sinora riconosciuti dalla critica. Ma non meno simbolico è che il primo fototesto italiano si riveli nient'altro che un plagio.

\section{Bibliografia}

Albertazzi, Silvia, Letteratura e fotografia, Roma, Carocci, 2017.

Amiel, Henri-Fréderic, Diario intimo [I884-I927], a cura di P. Mensi, Ravenna, Longo, 2000.

Angeli, Diego, Linarrivabile, Roma, Bontempelli, I89I.

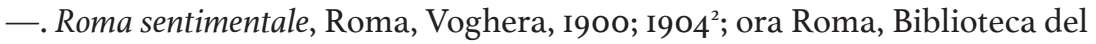
Vascello, I990.

- Le chiese di Roma, Roma, Società Editrice Dante Alighieri, I90o.

- Liliana Vanni, Catania, Giannotta, I900.

- Römische Stimmungsbilder, trad. di E. Müller-Röder, Leipzig, Rothbarth, [s.d., ma I905].

-. Le cronache del Caffè Greco, Milano, Treves, I930; ora, a cura di S. Stringini, Roma, Bulzoni, 200I.

- Taccuini romani. I dipinti di Diego Angeli della collezione del Museo di Roma in Trastevere, a cura di S. Bonfili, Roma, Gangemi, 2020.

Baldini, Antonio - Moretti, Marino, Carteggio. I9I5-I962, a cura di E. Colombo, Roma, Edizioni di Storia e Letteratura, 1997.

Barthes, Roland, La camera chiara [1980], trad. di R. Guidieri, Torino, Einaudi, I980.

Bigongiari, Piero, «Moretti tra il sole e la morte» [I977], in Id., Poesia italiana del Novecento, tomo I, La prima generazione, Milano, il Saggiatore, I978, pp. 45-63.

Carrara, Giuseppe, Storie a vista. Retorica e poetiche del fototesto, Milano - Udine, Mimesis, 2020.

Comberiati, Davide, Nessuna città d'Italia è più crepuscolare di Roma. Le relazioni fra il cenacolo romano di Sergio Corazzini e i simbolisti belgi, Bruxelles et al., Peter Lang, 20I4.

Cometa, Michele, «Forme e retoriche del fototesto letterario», in Id. - R. Coglitore (a cura di), Fototesti. Letteratura e cultura visuale, Macerata, Quodlibet, 20I6, pp. 69-II5.

tornava sul sepolcro del grande amante» (132-133; segue una citazione da The Magnetic Lady to Her Patient di Shelley, appunto; ma «Jane» si chiamava, come si ricorderà, pure la sventurata deuteragonista di Bruges-la-Morte). 
Contorbia, Franco, «I cigni di Bruges (per una "fonte” morettiana)», in Per Marino Moretti, numero monografico de Il lettore di provincia, XI, 4I-42, aprile-settembre i980, pp. 63-72.

-. «Diego Angeli», in F. Contorbia (a cura di), Giornalismo italiano, II: I9oII939, Milano, Mondadori, 2007, pp. 32-36.

Cortellessa, Andrea, «Bruges-la-Morte, o della città-montaggio», in S. Chiodi

- D. Giglioli (a cura di), Linee di montaggio, numero monografico de il verri, LXII, 68, ottobre 20I8, pp. 86-IIO.

—. «Bruges-la-Morte, Nadja, Vertigo. Psicologia di tre città», in M. Vallora (a cura di), Dal nulla al sogno. Dada e Surrealismo dalla Collezione del Museo Boijmans Van Beuningen, catalogo della mostra di Alba, 27 ottobre 2018 28 febbraio 20I9, Cinisello Balsamo, Silvana, 20I8, pp. 334-343.

D’Anna, Riccardo, «Roma preraffaellita. Note su Gabriele D’Annunzio, Diego Angeli, Giulio Aristide Sartorio», Atti della Accademia nazionale dei Lincei. Classe di scienze morali, storiche e filologiche. Memorie, 9, 1996, pp. 256-364.

—. Diego Angeli narratore. In margine a «Roma preraffaellita», Roma, Editoriale dell'Urbe, 1998.

—. «Primoli, Giuseppe Napoleone», ad $v$. in Dizionario Biografico degli Italiani, vol. 85, Roma, Istituto della Enciclopedia Italiana, 20I6, https://treccani. it/enciclopedia/giuseppe-napoleone-primoli_\%28Dizionario-Biografico\%29/ (consultato il 26.06.202I).

Edwards, Paul, «Les reliques [1997]», in Id., Soleil noir. Photographie et littérature: des origines au surréalisme, Rennes, Presses Universitaires de Rennes, 2008.

Faitrop Porta, Anne-Christine, La letteratura francese nella stampa romana (I880-I90o). Studio e bibliografia, Napoli-Roma, Edizioni Scientifiche Italiane, 1992.

Falzone del Barbarò, Michele, «Dieci anni di fotografia a Roma (I880-I890)», in Ghidetti 1979: 245-252.

Farini, Claudia, «Intertestualità crepuscolare; Gozzano e Rodenbach», Lingua e Stile, XXVII, 3, settembre I992, pp. 437-446.

—. «Georges Rodenbach e Marino Moretti: tra simbolismo e crepuscolarismo», in Livi-Ricci 2002, pp. I57-I68.

Ghidetti, Enrico (a cura di), Roma bizantina, con una nota di M. Falzone del Barbarò, Milano, Longanesi, 1979.

Govoni, Corrado, Armonia in grigio et in silenzio, Firenze, Lumachi, 1903.

-. Armonia in grigio et in silenzio, a cura di L. Barile, Milano, Scheiwiller, I989.

-. Armonia in grigio et in silenzio, a cura di A. Scarano, Bari, Palomar, 1992.

Grojnowski, Daniel, «L'invention du récit-photo. Bruges-la-Morte de Georges Rodenbach», in P. Mourier-Casile - D. Moncond'huy (a cura di), L'image 
génératrice de textes de fiction, numero monografico di La Licorne, 35, 1995, pp. I27-I38 (non ripreso in Id., Photographie et langage. Fictions illustrations informations visions théories, Paris, Josè Corti, 2002).

Guglielminetti, Marziano, «I fiamminghi, devozione e straniamento», in Livi-Ricci 2002, pp. I39-I45.

Livi, François, Dai simbolisti ai crepuscolari, Milano, Istituto di Propaganda Libraria, 1974.

-. "Govoni e i poeti di lingua francese», in A. Folli (a cura di), Corrado Govoni, Atti delle giornate di studio di Ferrara, 5-7 maggio 1983, Bologna, Cappelli, 1984, pp. 5I-I05.

-. La parola crepuscolare. Corazzini, Gozzano, Moretti, Milano, Istituto di Propaganda Libraria, 1986.

—. «Marino Moretti e il mito di Bruges», in Livi-Ricci 2002, pp. I47-I56.

Livi, François - Ricci, Manuela (a cura di), Itinerari europei di Marino Moretti (I885-1979), numero monografico di Revue des études italiennes, n.s., XLVIII, I-2, janvier-juin 2002.

Lombardi, Laura, «Il paesaggio "stato d'animo" nella seconda metà del XIX secolo: verso una "georgica dello spirito"», in C. Sisi (a cura di), La pittura di paesaggio in Italia. L'Ottocento, Milano, Electa, 2003, pp. 63-75.

Martini, Fausto Maria, Si sbarca a New York, Milano, Mondadori, 1930; ora, a cura di G. Baldassarri, Roma, Salerno, 2008.

Moretti, Marino, Poesie 1905-19I4, Milano, Treves, I9I9; ora, a cura di R. Cremante, Milano, La nave di Teseo, 2019.

-. La Casa del Santo Sangue, prefazione di G.A. Borgese, Milano, Mondadori, 1930.

—. «Bruges la risvegliata», in Corriere della Sera, 25 agosto 1938.

-. Tutte le poesie seguite da tre idilli in prosa, Milano, Mondadori, 1966.

Moretti, Marino - Palazzeschi, Aldo, Carteggio, I, 1904-I925, a cura di S. Magherini, Roma, Edizioni di Storia e Letteratura - Firenze, Università degli studi di Firenze, 1999.

Negro, Silvio, «Primoli fotografi in Roma», in Id., Roma non basta una vita, prefazione di E. Cecchi, Vicenza, Neri Pozza, 1962; ora, con introduzione di S. Malatesta, ivi, 2016, pp. 330-334.

Nieddu, Maria Elisabetta, «Da Bruges-la-Morte a Le Mirage. La perdita di un personaggio?», in L. Hutcheon - M. Fusillo - M. Guglielmi (a cura di), L'adattamento: le trasformazioni delle storie nei passaggi di codice, numero monografico di Between, II, 4, 20I2, http://ojs.unica.it/index.php/between/ article/view/635/566 (consultato il 26.06.202I).

Oliva, Gianni, «"Cuore bizantino". Società e moda in Diego Angeli» [2002], in Id., Centri e periferie. Particolari di geo-storia letteraria, Venezia, Marsilio, 2006, pp. I09-II6. 
Praz, Mario, «Roma sentimentale» [I940], in Id., Motivi e figure, Torino, Einaudi, I945, pp. II3-II6; poi, col titolo «La Roma dannunziana», in Id., Il patto col serpente. Paralipomeni di "La carne, il morte e il diavolo nella letteratura romantica", introduzione di G. Macchia, Milano, Mondadori, I972; ora Milano, Leonardo, I995, pp. 329-333.

Robaey, Jean, «Moretti e Rodenbach», Il lettore di provincia, XXV, 88, dicembre I993, pp. 3I-47.

Rodenbach, Georges, Bruges-la-Morte, Paris, Flammarion, I892; ora, a cura di J.-P. Bertrand e D. Grojnowski, ivi, 1998.

—. Bruges la morta, trad. di F.M. Martini, Roma, Voghera, I907.

—. Bruges la morta, a cura di P. Décina Lombardi, Milano, Mondadori, 1997.

Romagnoli, Sergio, «Marino Moretti pellegrino nelle Fiandre», in G. Calisesi (a cura di), Marino Moretti, Atti del convegno di Cesenatico, ottobre I975, Milano, il Saggiatore 1977, pp. 76-88.

Russo, Luigi, I narratori, Roma, Leonardo da Vinci, I923; ora, a cura di G. Ferroni, Palermo, Sellerio, 1987.

Sanguineti, Edoardo, "Govoni tra liberty e crepuscolarismo» [I984], in Id., La missione del critico, Genova, Marietti, I987, pp. 2 I-49.

Targhetta, Francesco, Corrado Govoni 1903-1907, tesi di dottorato, relatori F. Brugnolo e G. Baldassarri, Università di Padova, 2008.

—. «La nebbia dentro l'anima di Bruges, capitale di tutti i crepuscolari», Corriere della Sera, suppl. «La Lettura», I9 giugno 2016.

Valeri, Diego, Guida sentimentale di Venezia, Padova, Le Tre Venezie, I942; ora Torino, Lindau, 2020.

Veneruso, Danilo, «Angeli, Diego», ad v. in Dizionario Biografico degli Italiani, vol. 3, Roma, Istituto della Enciclopedia Italiana, I96I, https://www.treccani.it/enciclopedia/diego-angeli_(Dizionario-Biografico)/ (consultato il 26.06.202I).

Villa, Angela Ida, Neoidealismo e rinascenza latina tra Otto e Novecento. La cerchia di Sergio Corazzini. Poeti dimenticati e riviste del crepuscolarismo romano (I903-I907), Pisa-Roma, Istituti Editoriali e Poligrafici Internazionali, I999.

Violato, Gabriella, Bibliographie de Rodenbach et Semain en Italie, Paris-Firenze, Didier-Sansoni, 1965. 